\title{
The Desirability Optimization Methodology; a Tool to Predict Two Antagonist Responses in Biotechnological Systems: Case of Biomass Growth and Hyoscyamine Content in Elicited Datura starmonium Hairy Roots
}

\author{
Ryad Amdoun ${ }^{1,2}$, Lakhdar Khelifi ${ }^{1 *}$, Majda Khelifi-Slaoui ${ }^{1}$, Samia Amroune ${ }^{1}$, Mark Asch ${ }^{3}$, Corinne \\ Assaf-Ducrocq ${ }^{4}$, Eric Gontier ${ }^{4}$ \\ ${ }^{1}$ Laboratoire des Ressources Génétiques et Biotechnologie, École Nationale Supérieure Agronomique (ES1603), 16200 El-Harrach, Algiers - Algeria. \\ ${ }^{2}$ Laboratoire des cultures in vitro, Institut National de la Recherche Forestière (INRF), 37 Bp chéraga Bainem, Algiers - Algeria. \\ ${ }^{3}$ Université de Picardie Jules Verne, UFR des Sciences, Laboratoire Amiénois de Mathématique Fondamentale et Appliquée, CNRS-UMR 6140, 33 rue \\ Saint Leu, 80039 Amiens Cedex 1 - France. \\ ${ }^{4}$ Unité de Recherche EA3900 BIOPI-UPJV Biologie des plantes et Innovation, Université de Picardie Jules Verne, UFR des Sciences, Ilot des poulies, 33 \\ rue Saint Leu, 80039 Amiens Cedex 1 - France.
}

"Corresponding author: Lakhdar Khelifi, Laboratoire des Ressources Génétiques et Biotechnologie, École Nationale Supérieure Agronomique (ES1603), 16200 El-Harrach, Algiers - Algeria. Tel/Fax:+213- 23828504, E-mail: 1.khelifi@ensa.dz

Received: 11 Sep. 2015; $\quad$ Revised: 2 Dec. 2017; Accepted: 11 Mar. 2018; Published online: 18 Apr. 2018

\begin{abstract}
Background: The use of the desirability function approach combined with the response surface methodology (RSM), also called Desirability Optimization Methodology (DOM), has been successfully applied to solve medical, chemical, and technological questions. It is particularly efficient for the determination of the optimal conditions in natural or industrial processes involving different factors leading to the antagonist responses.

Objectives: Surprisingly, DOM has never been applied to the research programs devoted to the study of plant responses to the complex environmental changes, and thus to biotechnological questions.

Materials and Methods: In this article, DOM is used to study the response of Datura stramonium hairy roots (HRs), obtained by genetic transformation with Agrobacterium rhizogenes $\mathrm{A}_{4}$ strain, subjected to the jasmonate treatments.

Results: Antagonist effects on the growth and tropane alkaloid biosynthesis are confirmed. With a limited number of experimental conditions, it is shown that $0.06 \mathrm{mM}$ jasmonic acid (JA) applied for $24 \mathrm{~h}$ leads to an optimal compromise. Hyoscyamine levels increase by up to $290 \%$ after $24 \mathrm{~h}$ and this treatment does not significantly inhibit biomass growth.

Conclusions: It is thus demonstrated that the use of DOM can efficiently - with a minimized number of replicates - leads to the optimization of the biotechnological processes.

Keywords: Desirability Optimization Methodology (DOM); Hyoscyamine; Jasmonic Acid; Multiresponse Optimization; Response Surface Methodology (RSM).
\end{abstract}

\section{Background}

When using a complex system, several experimental factors have to be optimized so it is essential to evaluate alternative analytical procedures according to multiple criteria $(1,2)$. The determination of the optimum conditions for the input variables requires the simultaneous consideration of all responses. This is called a multiresponse problem $(3,4)$ based on Multicriteria Decision Making. To obtain a satisfactory compromise, the desirability approach is used as a powerful tool in multi-response systems (4). The desirability function, defined by Harrington (1965) (5) and by Derringer and Suich (1980) (3), is one of the approaches used for factor optimization in such systems (6-8). It is based on the transformation of all the obtained responses from different scales into a scale-free value. The values of desirability functions lie between 0 and 1 . The value 0 is attributed when the factors give an undesirable response, while the value 1 corresponds to the optimal performance for the studied factors $(3,4)$.

Copyright (C 2017 The Author(s); Published by National Institute of Genetic Engineering and Biotechnology. This is an open access article, distributed under the terms of the Creative Commons Attribution-NonCommercial 4.0 International License (http://creativecommons.org/licenses/ by-nc/4.0/) which permits others to copy and redistribute material just in noncommercial usages, provided the original work is properly cited. 
As an example in biological sciences, an optimization strategy based on the desirability function approach has been used for the microbial glutamine production (9). Furthermore, for studying the behavior of the complex systems, the use of the desirability function approach combined with the classic experimental designs enables the following matters (10-12):

A reduction in the number of modalities to be testedand a determination of the optimal compromise betweendifferent levels of environmental factors leading to theantagonist responses.

Among the classic experimental designs, Response Surface Methodology (RSM) is widely used because of its simplicity and effectiveness. The goal of RSM is to explore the relationship between the response (a dependent variable) and the studied factors (an independent variable) involved in an experiment. The mathematical model of RSM is a polynomial equation (Eq.) of the second degree which has the advantage of being easy to estimate and then to apply for the approximation of the response (13-15).

The combined application of RSM and the desirability approach gives rise to a more powerful method for finding an optimal balance between the responses, including antagonist responses (multicriteria optimization). This combination of the RSM and desirability function has resulted in a new method called "Desirability Optimization Methodology or DOM" (16). Although quite commonly used in industrial microbiology, biochemistry, and chemical analysis ( 7 , $10,17)$, to the best of our knowledge, such a method has never been applied to the plant biotechnological studies and, more specifically, to the study of the in vitro response of a plant secondary metabolism to the environmental factors.

Because of the interest in the biotechnological production of the plant secondary metabolites, we chose to evaluate the potential of DOM for studying the metabolic changes induced by elicitation in the hairy roots (HRs) of Datura stramonium. As widely described (18-20), hairy root cultures of Datura species produce tropane alkaloids. Their biosynthesis can be modulated by the culture medium composition or by elicitor treatments. Such treatments can lead to tropane alkaloid levels as high as, and even higher than those measured in the whole plants. Because of their rapid growth capacity, HRs are also a good experimental system.

Synthesized from linolenic acid, jasmonate is a signal molecule involved in the response to the different kinds of stress, and more specifically, to the elicitation; it thus leads to the plant defense reactions (21-24). It stimulates the accumulation of the secondary metabolites such as tropane alkaloids in solanaceous plants (25). The use of elicitors or phytohormones, such as jasmonate and its volatile derivative methyl jasmonate $(26,27)$, is a possible approach for increasing tropane alkaloid biosynthesis in Datura species $(25,28)$. However, plant growth is then inhibited $(25,29,30)$. This kind of antagonist response is often observed during stress (31$35)$. In order to develop an efficient in vitro technology to produce secondary metabolites using elicitation, optimum conditions must be determined i.e. elicitor concentration and elicitation time and duration. In Datura hairy root cultures, because growth decreases when alkaloid levels increase, determining an optimal treatment condition thus becomes a difficult challenge (36-38).

\section{Objectives}

This study investigates how efficiently DOM can be used to study the antagonist response (growth vs. alkaloid synthesis) of Datura stramonium HRs subjected to jasmonate treatments. Subsequently, i) elicitation conditions are optimized for hyoscyamine production and ii) this mathematical modeling using DOM may serve as an example for other studies devoted to the plant biology and biotechnology.

\section{Materials and Methods}

\subsection{Plant Material}

The HRs of Datura stramonium, obtained by genetic transformation with Agrobacterium rhizogenes $\mathrm{A}_{4}$ strain on hypocotyls, were examined for their performance: growth (dry weight), alkaloid content, and reaction to elicitation $(14,20)$. Then, the best hairy root line was selected and subcultured every $20-25^{\text {th }}$ day in fresh B5 Gamborg hormone-free culture medium containing 3\% sucrose (35). All hairy root cultures were grown at 26 $\pm 1{ }^{\circ} \mathrm{C}$ in the dark. For experiments, root tips $(0.3 \mathrm{~g}$ fresh weight) were cultured in Petri dishes containing $20 \mathrm{~mL}$ of hormone-free medium (Gamborg) (39) with $3 \%$ sucrose.

\subsection{Elicitation}

The solution of jasmonic acid (JA) [(-)jasmonic acid from Sigma-Aldrich] was prepared by dissolving jasmonic acid in an adequate volume of ethanol. It was then filtered through a membrane filter (pore size: 0.2 $\mu \mathrm{m}$ Nalgene) (14). Five concentrations of the jasmonic acid $(J A): 0.0172,0.0250,0.0500,0.0750$, and 0.0830 $\mathrm{mM}$ and five exposure times $(E T): 1.6,8,28,48$, and 54.4 hours were tested according to CCDO, (see section RSM: experimental design). The choice of concentrations and ETs was based on a literature study $(25,30)$ and on our previous works. The elicitation experiments were initiated after the transfer of roots to 
the fresh B5 medium $(20 \mathrm{~mL})$ on the $12^{\text {th }}$ day of culture (logarithmic phase of growth according to Amdoun et al., 2009 (20).

All cultures were grown in Petri dishes containing $20 \mathrm{~mL}$ of B5 medium and in darkness at $26 \pm 1{ }^{\circ} \mathrm{C}$. The control (ethanol-water solution without JA) was the same hairy root line in the same conditions of the culture. All culture experiments were carried out in the experimental setup design of triplicates.

\subsection{Determination of Biomass and Hyoscyamine Content}

Applying a precision balance, the biomass of the correspondent dry weight of HRs, obtained after oven drying for $48 \mathrm{~h}$ at $40^{\circ} \mathrm{C}$, was measured. The alkaloids were extracted using a method described by Amdoun et al. (2009) (20). Hyoscyamine was analyzed by the GC-MS method previously reported by Kartal et al. (2003) (40).

\subsection{RSM: Experimental Design}

The CCDO, described by Box and Wilson (1951) (13), was chosen for this work. It is composed of a factorial design $2^{\mathrm{k}}$, with at least one experimental central point and a star designs whose axial points: $-\alpha$ and $+\alpha$ are placed on the axis of each factor. The $E T$ and jasmonic $J A C$ experiments which influence the biomass and hyoscyamine content were carried out according to the CCDO. Only fourteen experiments $\left(\mathrm{R}_{1}\right.$ to $\left.\mathrm{R}_{14}\right)$ were necessary to model the $J A C$ and $E T$ influence on biomass and alkaloid production. The CCDO, the variables $(J A C ; E T)$ and their studied levels (5 concentrations and 5 elicitation times) are presented in Figure 1. Each point $\left(\mathrm{R}_{1} \ldots \mathrm{R}_{14}\right)$ corresponds to one experiment. The points $R_{1}$ to $R_{4}$ represent the factorial design. The points $\mathrm{R}_{5}$ to $\mathrm{R}_{8}$ are the star design. The points $\mathrm{R}_{9}$ to $\mathrm{R}_{14}$ represent the experiments carried out in the central experimental design. Six repetitions $\left(\mathrm{R}_{9}\right.$ to $\left.\mathrm{R}_{14}\right)$ were performed and the optimal criterion was orthogonality. In this case, $\pm \alpha$ $= \pm 1.32$ was chosen respect to the criterion.

The levels of variable $X_{i}$ were coded from the equation $X_{i}=\left(A_{i}-A_{0}\right) / \Delta A_{i}$, where $X_{i}$ is the independent variable coded value, $A_{i}$ is the independent variable real value, $A_{0}$ is the independent variable real value on the central point, and $\Delta A_{i}$ is the step change value.

\subsection{Expression of Measured Responses and Results}

The biomass growth was represented by a growth index (GI) calculated from equation 1 . For a GI $=1$, hairy root growth was not affected by JA. On the other hand, a GI $<1$ has revealed an affected growth. At the same time, the increase in hyoscyamine content after elicitation was represented by the elicitation index (EI). It was

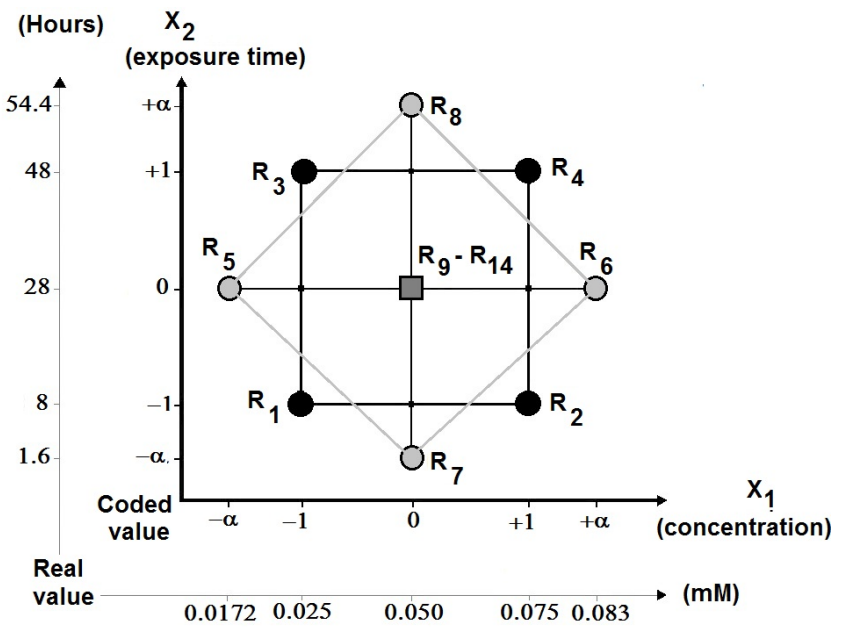

Figure 1. The central composite design orthogonal (CCDO): variables and their studied levels. Each point corresponds to one experiment. The points $\mathrm{R}_{1}$ to $\mathrm{R}_{4}$ are the factorial design, the points $R_{5}$ to $R_{8}$ are the star design, the points $R_{9}$ to $R_{14}$ are the experiments carried out in the central experimental design (with coded value $\pm \alpha= \pm 1.32$ for the orthogonality criterion).

calculated from equation 2 . If the hyoscyamine content was increased, the EI was $>1$.

$G I=\frac{\text { biomass of HR elicited }\left(g D W \cdot l^{-1}\right)}{\text { biomass of HR controls }\left(g D W \cdot l^{-1}\right)}$

(Eq.1)

$E I=\frac{\text { specefic production of hyoscyamine }\left(g \cdot g^{-1} D W\right) \text { of HR elicited }}{\text { specefic production of hyoscyamine }\left(g \cdot g^{-1} D W\right) \text { of HR controls }}(E q .2)$

3.6. Mathematical Modeling of Measured Responses: Growth Index $\left(Y_{G Y}\right)$ and Elicitation Index $\left(Y_{E}\right)$

The results (i.e., the measured GI and EI) obtained from the experiments defined in Figure 1 (CCDO) were used for the mathematical model expression of the effects of $J A C$ and elicitation time (ET) on GI and EI. The results of the CCDO were used for the computation of the coefficients of the two quadratic models Eq. 3 and Eq. 4 by the method of the least squares (41).

$$
\begin{aligned}
& Y_{G I}=\alpha_{0}+\alpha_{1} J A C+\alpha_{2} E T+\alpha_{11} J A C^{2}+\alpha_{22} E T^{2}+\alpha_{12} J A C E T \\
& Y_{E I}=\alpha_{0}+\alpha_{1} J A C+\alpha_{2} E T+\alpha_{11} J A C^{2}+\alpha_{22} E T^{2}+\alpha_{12} J A C E T \quad \text { (Eq. 4) }
\end{aligned}
$$

Where, $Y_{G I}$ and $Y_{E I}$ are the responses (biomass or hyoscyamine level), $J A C$ is the jasmonic acid concentration, ET is the elicitation time, and $\alpha_{0}, \alpha_{1} \ldots \alpha_{12}$ are the model's coefficients. After the overall evaluation $\left(R^{2}\right.$, ANOVA) followed by adjustment of the model, they were used for the simultaneous optimization of $Y_{G I}$ and $Y_{E I}$ with the desirability function approach. 


\subsection{Desirability Function}

The desirability function approach transforms an estimated response into a scale-free value, called desirability $(3,4,5)$. The objectives of optimization can be used to maximize, minimize, or obtain the target value of the response (42). Depending on the objective different desirability functions can be employed. Derringer and Suich (1980) (3) applied a special function for the transformation of the $Y_{i}$ responses to the desirability $d_{i}\left(Y_{i}\right)$. So, two transformations are proposed:

The one-sided transformation used to maximizeor minimize Yi; the two-sided transformation (equation 5) usedto obtain the target value ti for Yi where li and ui are thelower and upper bounds on the studied response (suchthat li $<$ ti $<$ ui).

The powers $s$ and $t$ correspond to the weighted factor. $s$ and $t$ are the parameters that determine the shape of $d_{i}\left(Y_{i}\right)$. For $s=t=1$, the desirability function increases linearly to $t_{i}$. If $s<1$ and $t<1$, the function is concave and if $s>1$ and $t>1$, the shape is convex $(4,42)$.

$$
d_{i}\left(Y_{i}\right)= \begin{cases}0, & Y_{i}<l_{i}, \\ \left(\frac{Y_{i}-l_{i}}{t_{i}-l_{i}}\right)^{s}, & l_{i} \leq Y_{i} \leq t_{i}, \\ \left(\frac{Y_{i}-u_{i}}{t_{i}-u_{i}}\right)^{t}, & t_{i} \leq Y_{i} \leq u_{i}, \\ 0, & Y_{i}>u_{i}\end{cases}
$$

The overall desirability function $D$ is defined as the geometric average of the individual desirability functions of each response $d_{i}\left(Y_{i}\right)$ (equation 6) where $n$ is the number of responses. The optimal solutions are determined by maximizing $D(4)$.

$$
D=\left(\prod_{i=1}^{n} d_{i}\left(Y_{i}\right)\right)^{\frac{1}{n}}
$$

\subsection{Statistical Analyses}

ANOVA (Fisher Test) was used for an overall evaluation of the models. Tests at 5\% risk were considered significant. The coefficient of determination $R^{2}$ has to be taken into account. It represents the ratio of the variance suggested by the mathematical model with respect to the overall variance and varies from 0 to 1 . It was calculated from equation 7 where $S C E$ is the sum of the squares of the residuals and $S C T$ is the total sum of the squares. It is also necessary to take into account the Absolute Average Deviation (AAD), whose value must be low. The $R^{2}$ analysis alone is not a measure of the model's accuracy even if the value is large. The AAD is calculated by the equation 8 where $Y_{i, m e s}$ and $Y_{i, p r e}$ are the measured and predicted responses, respectively, and $n$ is the number of the experimental run. The simultaneous evaluation of these two values $\left(R^{2}\right.$ and $\left.\mathrm{AAD}\right)$ shows the overall predictive capability of the mathematical model (43).

$$
\begin{aligned}
R^{2} & =\frac{S C E}{S C T} \\
A A D & =\left\{\left[\sum_{i=1}^{n}\left(\left|Y_{i, \text { mes }}-Y_{i, p r e}\right| / Y_{i, p r e}\right)\right] / n\right\} \times 100
\end{aligned}
$$

\section{Results}

\subsection{Modeling the Growth Index $\left(Y_{G I}\right)$ and Elicitation Index $\left(Y_{E I}\right.$}

The results obtained after the experiments according to the CCDO were used to compute by the least squares method; the coefficients of the two quadratic models (equations 3 and 4 ) are expressing the effects of $J A C$ ET on GI and EI.

The obtained two quadratic models $Y_{G I}$ and $Y_{E I}$ show large coefficients of determination $\left(R^{2}=0.80\right.$ for $Y_{G I}$ and $R^{2}=0.90$ for $\left.Y_{E I}\right)$ with values $<10 \%$ for AAD $\left(6.5 \%\right.$ for $Y_{G I}$ and $6.0 \%$ for $\left.Y_{E I}\right)$. Thus, these two models explain $80 \%$ and $90 \%$, respectively, of the $Y_{G I}$ and $Y_{E I}$ variability; they are predictive overall. Following the variance analysis (Table 1), evaluation and diagnostic of these two models, all the insignificant terms were removed $(14,44)$.

In this way, the adjusted mathematical models became:

$\hat{Y}_{G I}=0.72-0.11 J A C-0.14 E T+0.01 E T^{2}$

$\hat{Y}_{E I}=1.76-0.30 J A C-0.60 E T+0.25 J A C^{2}+0.22 E T^{2}$

\subsection{Simultaneous Optimization by the Desirability Function Approach}

Equation 5 was used for the two-sided transformation of the responses to an individual desirability scale where they increased linearly from 0 to 1 . They were noted $d_{G I}$ for GI and $d_{E I}$ for elicitation index (EI). Equation 6 led to the overall desirability function $D$ defined to find an optimal solution for both $J A C$-ET factors. Figures 2 and $\mathbf{3}$ show the response surface and the contour plot of the desirability function in the space of the factor $J A C$ and ET for the two models.

For biomass, the maximum desirability values are: 0.7 for GI (target values for GI), between small values of $E T$ 
Table 1. Analysis by ANOVA for the quadratic models of the growth and elicitation indexes. The results in bold are significant at $5 \%$ risk: $*$ significant; $* *$ highly significant; $* * *$ very highly significant.

\begin{tabular}{lcccccc}
\hline Source & Sum of Squares & df & Mean Square & F-value & p-value & Significance \\
\hline Growth Index (GI) & & & & & & \\
\hline Model & 0.304 & 5 & 0.060 & 8.753 & $\mathbf{0 . 0 0 4}$ & $* *$ \\
$J A C$ & 0.084 & 1 & 0.084 & 12.106 & $\mathbf{0 . 0 0 8}$ & $* *$ \\
$E T$ & 0.150 & 1 & 0.150 & 21.661 & $\mathbf{0 . 0 0 1}$ & $* *$ \\
$J A C x E T$ & 0.010 & 1 & 0.010 & 1.437 & 0.264 & \\
$J A C^{2}$ & 0.001 & 1 & 0.001 & 0.261 & 0.623 & \\
$E T^{2}$ & 0.057 & 1 & 0.057 & 8.297 & $\mathbf{0 . 0 2 0}$ & $*$ \\
Residual & 0.055 & 8 & 0.006 & & & \\
Cor Total & 0.360 & 13 & & & & \\
\hline Elicitation Index (EI) & & & & & & \\
\hline Model & 4.014 & 5 & 0.802 & 15.560 & $\mathbf{0 . 0 0 0}$ & $* * *$ \\
$J A C$ & 0.655 & 1 & 0.655 & 12.714 & $\mathbf{0 . 0 0 7}$ & $* *$ \\
$E T$ & 2.686 & 1 & 2.686 & 52.067 & $\mathbf{0 . 0 0 0}$ & $* * *$ \\
$J A C x E T$ & 0.002 & 1 & 0.002 & 0.048 & 0.831 & \\
$J A C^{2}$ & 0.375 & 1 & 0.375 & 7.278 & $\mathbf{0 . 0 2 7}$ & $*$ \\
$E T^{2}$ & 0.293 & 1 & 0.293 & 5.695 & $\mathbf{0 . 0 4 4}$ & $*$ \\
Residual & 0.412 & 8 & 0.051 & & & \\
Cor Total & 4.427 & 13 & & & & \\
\hline
\end{tabular}

Cor Total: Corrected Total sum of squares

and mean values of $J A C$. The desirability is high for $\mathrm{EI}=$ 2.15 (target values for $\mathrm{EI})\left(d_{E I}=1\right)$, even at low $J A C$ (Fig. 3). The optimal solutions fit with all the intersection points between $J A C$ and $E T$ and correspond to the values of the maximized desirabilities for GI and EI, so $d_{G I}=1$ and $d_{E I}$ $=1$, respectively (Figs. 2 and 3). These two responses, individually, show an area of optimal solutions for $d i=$ 1. The level of difficulty of multiresponse optimization increases when the optimal areas of responses are too distant and do not cross. It is therefore necessary to find an optimal compromise between all the responses taken into consideration. Thus, the overall desirability function $D$ is the most used and significant approach (45).

Figure 4 shows the resulting overall desirability $D$ with respect to $J A C$ and $E T$. The area of optimal solutions decreases when the two responses GI and EI are taken simultaneously for the optimization.

This response surface analysis is due to the conflict of $J A C$ and $E T$ on the biomass and hyoscyamine level. So, the optimal combinations $J A C$ and $E T$ are presented by the intersection between $X_{1}$ and $X_{2}$ (red zone in Fig. 4) where $D=1$. The projected area corresponds to the optimal solutions (compromise) between the two antagonist responses (Fig. 4). This area has been defined with a limited number of experiments, thanks to the use of DOM. Thus, these results confirm the usefulness of DOM for finding the compromises between antagonist responses and, more specifically here, between hyoscyamine levels and the growth preservation.

To obtain the target values $\mathrm{GI}=0.7$ and $\mathrm{EI}=2.15$, one of the solutions which maximizes $D(=1)$ corresponds to 0.06 $\mathrm{mM}$ (coded 0.55 ) for $J A C$ and $23.2 \mathrm{~h}$ for ET (coded -0.24).

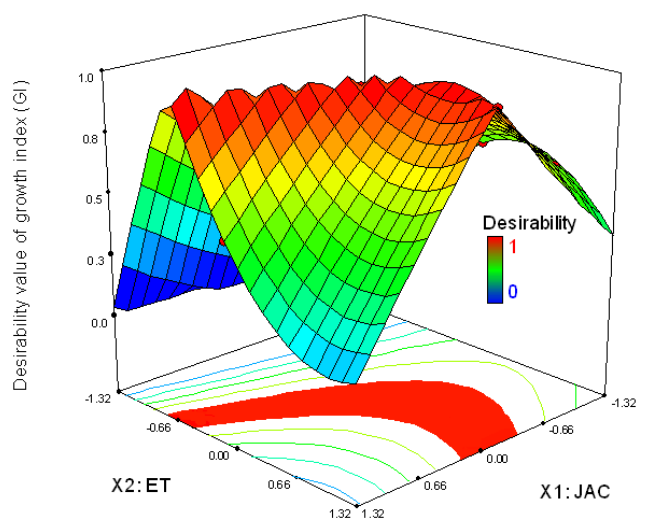

Figure 2. Response surfaces and estimated contours of the desirability value of the biomass as a function of JAC and elicitation time (ET). 


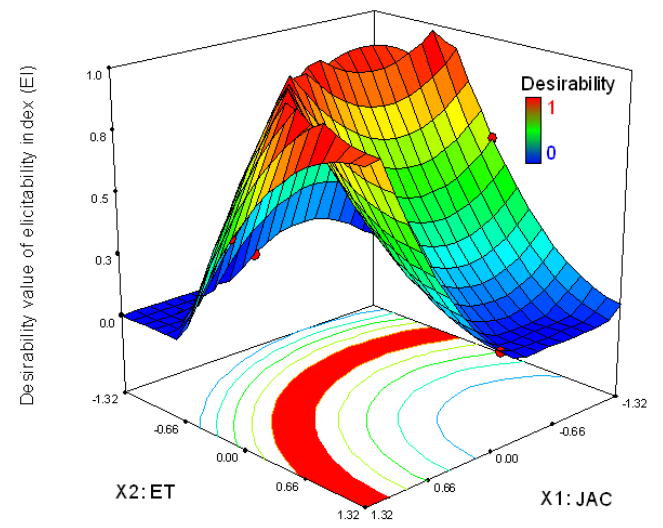

Figure 3. The response surfaces and estimated contours of the desirability value of hyoscyamine content as a function of $J A C$ and elicitation time $(E T)$.

\subsection{Optimization Levels by the Application of DOM}

To investigate the optimization level by DOM, a number of experiments were carried out with $J A C=0.06$ $\mathrm{mM}$ and $E T=23.2 \mathrm{~h}$ (Table 2). For practical reasons, the $E T$ was adjusted to $24 \mathrm{~h}$; the value that was included in the optimal solutions. Before elicitation experiments on the $12^{\text {th }}$ day of culture, the roots were transferred to a fresh B5 medium $(20 \mathrm{~mL})$. The responses on biomass and hyoscyamine level were studied at $24 \mathrm{~h}$ and $120 \mathrm{~h}$ after elicitation.

The obtained results show that JA elicitation $(0.06$ $\mathrm{M}$ for 24 hours) led to a 2.9-folds improvement in the hyoscyamine content $24 \mathrm{~h}$ after elicitation. However, the same hyoscyamine content was obtained in the control and elicited hairy roots $(E I=1.0) 120 \mathrm{~h}$ after elicitation. Nevertheless, the biomass was slightly affected $(\mathrm{GI}=0.9) 120 \mathrm{~h}$ after elicitation.

\section{Discussion}

\subsection{Modelization of $Y_{G I}$ and $Y_{E I}$}

For the $\hat{Y}_{G I}$ model, it can be seen that $J A C(-0.11)$ and ET (- 0.14) show a negative effect on hairy root growth (equation 9). So, if $J A C$-ET factors are increased, hairy root growth is inhibited (Fig. 5). In contrast, if $J A C-E T$ factors are low, hairy root growth is not inhibited.

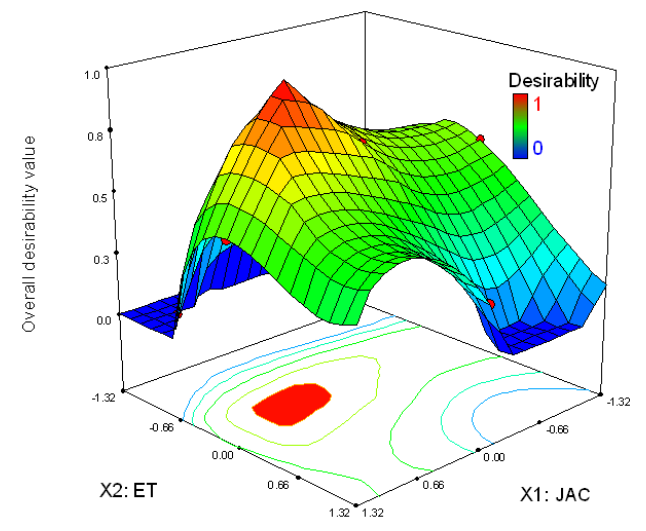

Figure 4. The response surfaces and estimated contours of the overall desirability as a function of $J A C$ and $E T$.

These results are similar to those obtained by other authors. For example, Kang et al. (2004) (30) reported that $1 \mathrm{mM}$ methyl jasmonate treatment did not have a negative effect on the growth of adventitious roots of Scopolia parviflora. However, when the adventitious root cultures were treated with $2.0 \mathrm{mM}$, the root GI decreased to $63 \%$ of the control culture. After $72 \mathrm{~h}$ of elicitation, the roots were severely damaged and died (Kang et al., 2004). Similar results have been reported by Deng (2005) (25) on the root growth inhibition of Datura stramonium using methyl jasmonate at a concentration of $>10^{-5} \mathrm{M}$.

An analysis of the $\hat{Y}_{E I}$ model (equation 10) shows a positive effect of JA $(+0.30)$ and a negative effect of $E T$ $(-0.60)$ on the hyoscyamine level. The effect of JA on the production of tropane alkaloids comes from the results which suggest that jasmonates regulate the expression of the key enzymes PMT (Putrescine N-Methyl Transferase) and $\mathrm{H} 6 \mathrm{H}$ (Hyoscyamine $6 \beta$-Hydroxylase) and the biosynthesis of the tropane alkaloids. The evaluation of jasmonate effects on the tropane alkaloid biosynthesis has been suggested by western blotting on adventitious root cultures of Scopolia parviflora (30). In Datura stramonium, methyl jasmonate was shown to increase phenylalanine levels, one of precursors of

Table 2. Optimization of the hyoscyamine content and biomass growth at 24 and $120 \mathrm{~h}$ after elicitation (results are rounded to one decimal point).

\begin{tabular}{llclcc}
\hline Jasmonic acid $(\mathbf{m M})$ & \multicolumn{2}{c}{ Hyoscyamine $\left(\mathbf{m g . g}^{-\mathbf{1}} \mathbf{D W}\right)$} & & \multicolumn{2}{c}{ Biomass $(\mathbf{g}$ DW.L } \\
\cline { 2 - 3 } \cline { 5 - 6 } & $24 \mathrm{~h}$ & $120 \mathrm{~h}$ & & $24 \mathrm{~h}$ & $120 \mathrm{~h}$ \\
\hline 0 (control) & $0.3 \pm 1.9$ & $6.3 \pm 0.2$ & & $5.0 \pm 0.2$ & $0.2 \pm 6.3$ \\
0.06 & $0.5 \pm 5.6$ & $6.5 \pm 0.2$ & & $0.2 \pm 5.0$ & $0.2 \pm 5.6$ \\
GI and EI & $\mathrm{EI}=2.9$ & $\mathrm{EI}=1.0$ & & $\mathrm{GI}=1.0$ & $\mathrm{GI}=0.9$ \\
\hline
\end{tabular}



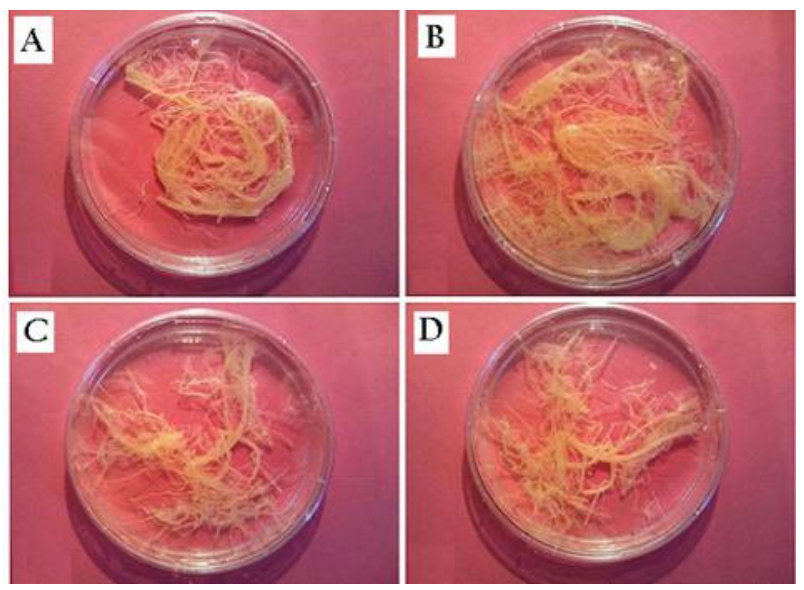

Figure 5. Elicited and control hairy root cultures. A: hairy root control on $13^{\text {th }}$ day of culture. B: hairy root control on $17^{\text {th }}$ day of culture. $\mathbf{C}$ : elicited hairy root culture on $13^{\text {th }}$ day of culture (0.0830 mM jasmonic acid; $24 \mathrm{~h}$ after elicitation). D: elicited hairy root culture on $17^{\text {th }}$ day of culture $(0.0830 \mathrm{mM}$ jasmonic acid; $120 \mathrm{~h}$ after elicitation).

tropane alkaloids, at $10^{-8} \mathrm{M}$. Subsequently, tropinone, tropine and $\varphi$-tropine levels were greatly increased by methyl jasmonate at $10^{-7}$ and $10^{-6} \mathrm{M}(25)$.

Thus, the two models: (9) and (10) confirm that JA is a sensitive elicitor with two antagonist responses: it increases the hyoscyamine content with growth inhibition of the HRs. So, a compromise between $J A C$ and $E T$ is essential in order to improve the hyoscyamine level with no inhibition on the root growth by using the desirability approach.

To obtain a satisfactory compromise, the optimal solution (JAC-ET combination) is defined by the target values $\left(t_{i}\right)$ of the each response. The average values $t_{i}$ of both bounds $l_{i}$ and $u_{i}$ are the target values for GI, $t_{i}=0.7$ $\left(l_{i}=0.4\right.$ and $\left.u_{i}=1\right)$, and for ET, $t_{i}=2.15\left(l_{i}=1.3\right.$ and $u_{i}=$ $3)$, respectively. The values of the bounds $l_{i}$ and $u_{i}$ of the GI and EI present the bounds of the experimental values obtained after application of the CCDO. To solve the conflict established between biomass and hyoscyamine content, the use of the average values as target values allows the solutions to be maximized and a satisfactory compromise to be established. The fitted models (9) and (10) were used for the simultaneous optimization of the $Y_{G I}$ and $Y_{E I}$ using the desirability function. In addition, the two-sided transformation was used in this work for multiresponse optimization and for both simultaneous responses.

\subsection{Application of DOM}

For various species like Scopolia parviflora (30) and Brugmansia suaveolens (46), the ET for the best production of hyoscyamine was 24 hours. The same authors have claimed the optimal $J A C$ varies from $0.01 \mathrm{mM}$ (30) to $2 \mathrm{mM}$ (46). Zabetakis et al. (1999) (26) have reported that $0.1 \mu \mathrm{M}$ of methyl jasmonate was the optimal concentration for the elicitation in the root culture of $D$. stramonium and increased the hyoscyamine production 1.8-fold compared to the control. In other species, Kang et al. (2004) (30) have reported the elicitation of the adventitious roots of Scopolia parviflora with $0.01 \mathrm{mM}$ with an elicitation time of $24 \mathrm{~h}$ increased the hyoscyamine content 1.8fold compared to the control over the same time without growth inhibition. In the same way, elicitation of the HRs of Brugmansia suaveolens by $2 \mathrm{mM} \mathrm{JA}$ for $24 \mathrm{~h}$ improved the yield of hyoscyamine 25 folds (46). However, these authors did not report the effect of $J A C$ on the biomass.

Several studies on jasmonic acid effects on the tropane alkaloid content and biomass growth show conflicting responses. It is to be expected that the experimental conditions which lead to an optimum for one of these factors are not the same as for another. So, the optimization of these two parameters is rather difficult. However, in this paper we have shown that DOM (i.e., RSM combined with the desirability approach) is a satisfactory compromise for multiresponse optimization. DOM is useful and easy to adapt to the complex biological systems such as hairy root cultures. In our case, it led to an optimal solution for the parameters $J A C$-ET with a 2.9-fold improvement in hyoscyamine content and a slight growth inhibition; $10 \%$ less than the control. It seems that $90 \%$ of the hairy root biomass is retained after 5 days of elicitation $(120 \mathrm{~h})$. Thus, the maximum overall desirability function is reached for: $J A C=$ $0.06 \mathrm{mM}$ and $E T=24$ hours. The present study was performed in a closed system, so the inhibitory effect of JA on biomass at low concentrations could be due to its prolonged presence in the culture medium. We can suppose that this effect will be absent in a system where the medium is changed regularly, such as a continuous or semi-continuous regime. In such a system, we anticipate that the high concentrations of JA (with a short ET) will not affect the biomass.

DOM has proved its efficiency for the development of the products in which different properties need to be associated (16). In this article we have shown that DOM is also extremely efficient for determining even antagonist responses in the plant biotechnological processes. These results thus show the way and opens up new opportunities for the other kinds of uses in the wide area of plant sciences and technologies. 


$\begin{array}{ll}\text { Abbreviations } \\ \text { B5 } & \text { Gamborg culture medium } \\ \text { SCE } & \text { Sum of the squares of residuals } \\ \text { SCT } & \text { Total sum of the squares } \\ Y E I & \text { Response of hyoscyamine content } \\ Y G I & \text { Response of growth level }\end{array}$

\section{Acknowledgements}

The authors gratefully acknowledge funding support from ENSA of Algiers, the Algerian Ministry of High Education and Research (MESRS), and the University of Picardie Jules Verne, Amiens, France.

\section{References}

1. Deming SN. Multiple-criteria optimization. J Chromatogr A. 1991;550:15-25. doi: 10.1016/s0021-9673(01)88527-7.

2. Hendriks MM, De Boer JH, Smilde AK, Doornbos DA. Multicriteria decision making. Chemometr. Intell Lab Syst. 1992;16(3):175-191. doi: 10.1016/0169-7439(92)80036-4.

3. Derringer G, Suich R. Simultaneous optimization of several response variables. J Quality Technol. 1980;12:214-219.

4. Jeong IJ, Kim KJ. An interactive desirability function method to multiresponse optimization. Eur J Oper Res.2009;195(2):412426. doi: 10.1016/j.ejor.2008.02.018.

5. Harrington ECJr. The desirability function. Ind Quality Control.1965;21:494-498.

6. Bourguignon B, Massart DL. Simultaneous optimization of several chromatographic performance goals using Derringer's desirability function. J Chromatogr A. 1991;586(1):11-20. doi: 10.1016/0021-9673(91)80019-d.

7. Safa F, Hadjmohammadi MR. Simultaneous optimization of the resolution and analysis time in micellar liquid chromatography of phenyl thiohydantoin amino acids using Derringer's desirability function. $J$ Chromatogr A. 2005;1078 (1-2):42-50. doi: 10.1016/j.chroma.2005.04.081

8. Candioti LV, De Zan MM, Cámara MS, Goicoechea HC. Experimental design and multiple response optimization. Using the desirability function in analytical methods development. Talanta. 2014;124:123-138. doi: 10.1016/j.talanta.2014.01.034.

9. Li J, Ma C, Ma Y, Li Y, Zhou W, Xu P. Medium optimization by combination of response surface methodology and desirability function: an application in glutamine production. Appl Microbiol Biotechnol. 2007;74(3):563-571. doi:10.1007/ s00253-006-0699-5.

10. Outinen K, Haario H, Vuorela P, Nyman M, Ukkonen E, Vuorela H. Optimization of selectivity in high-performance liquid chromatography using desirability functions and mixture designs according to prisma. Eur $J$ Pharmaceut Sci.1998;6(3):197-205. doi:10.1016/s0928-0987(97)10016-1.

11. Khodadoust S, Hadjmohammadi MR. Determination of $\mathrm{N}$-methylcarbamate insecticides in water samples using dispersive liquid-liquid microextraction and HPLC with the aid of experimental design and desirability function. Anal. Chim Acta. 2011;699(1):113-119. doi:10.1016/j.aca.2011.04.011.

12. Mourabet M, El Rhilassi A, El Boujaady H, Bennani-Ziatni M, El Hamri R, Taitai A. Removal of fluoride from aqueous solution by adsorption on Apatitic tricalcium phosphate using Box-Behnken design and desirability function. Appl Surf Sci. 2012;258(10):4402-4410. doi: 10.1016/j.apsusc.2011.12.125.
13. Box GEP, Wilson KB. On the experimental attainment of optimum conditions. J Roy Stat Soc B Stat Meth. 1951;13(1):145.

14. Amdoun R, Khelifi L, Khelifi-Slaoui M, Amroune S, Asch M, Assaf-Ducrocq C, Gontier E. Optimization of the culture medium composition to improve the production of hyoscyamine in elicited Datura stramonium L. hairy roots using the Response Surface Methodology (RSM). Int J Mol Sci. 2010;11(11):47264740. doi: 10.3390/ijms11114726.

15. Cui L, Wang ZY, Zhou XH. Optimization of elicitors and precursors to enhance valtrate production in adventitious roots of Valeriana amurensis Smir. ex Kom. Plant Cell Tiss Organ Cult. 2012;108(3):411-420. doi: 10.1007/s11240-011-0052-2.

16. Derringer GC. A Balancing Act: Optimizing a Product's Properties. Quality Prog. 1994;51-58.

17. Hu Z, Cai M, Liang HH. Desirability function approach for the optimization of microwave-assisted extraction of saikosaponins from Radix Bupleuri. Separ Purif Tech. 2008;61(3):266-275. doi: 10.1016/j.seppur.2007.10.016.

18. Nussbaumer P, Kapétanidis I, Christen P. Hairy root of Datura candida $\mathrm{x}$ aurea: effect of culture medium composition on growth and biosynthesis. Plant Cell Rep. 1998;17(5):405-409. doi: 10.1007/s002990050415.

19. Piñol MT, Palazón J, Cusidó RM, Ribó M. Influence of calcium ion-concentration in the medium on tropane alkaloid accumulation in Datura stramonium hairy roots. Plant Sci. 1999;141(1):41-49. doi: 10.1016/s0168-9452(98)00222-2.

20. Amdoun R, Khelifi L, Khelifi-Slaoui M, Amroune S, Benyoussef EH, Thi DV, Assaf-Ducrocq C, Gontier E. Influence of minerals and elicitation on Datura stramonium L. tropane alkaloid production: Modelization of the in vitro biochemical response. Plant Sci. 2009;177(2):81-87. doi: 10.1016/j. plantsci.2009.03.016.

21. Boitel-Conti M, Laberche JC, Lanoue A, Ducrocq C, SangwanNorreel B.S. Influence of feeding precursors on tropane alkaloid production during an abiotic stress in Datura innoxia transformed roots. Plant Cell Tissue Organ Cult. 2000;60(2):131-137. doi: 10.1023/a:1006426314274.

22. Yu LJ, Lan WZ, Qin WM, Xu HB. High stable production of taxol in elicited synchronous cultures of Taxus chinensis cells. Process Biochem. 2002;38(2):207-210. doi:10.1016/s00329592(02)00069-9.

23. Zhao J, Davis LC, Verpoorte R. Elicitor signal transduction leading to production of plant secondary metabolites. Biotechnol Adv. 2005;23(4):283-333. doi: 10.1016/j. biotechadv.2005.01.003.

24. Vasconsuelo A, Boland R. Molecular aspects of the early stages of elicitation of secondary metabolites in plants. Plant Sci. 2007;172(5):861-875. doi: 10.1016/j.plantsci.2007.01.006

25. Deng F. Effects of glyphosate, chlorsulfuron, and methyl jasmonate on growth and alkaloid biosynthesis of jimsonweed (Datura stramonium L.). Pestic Biochem. Physiol. 2005;82(1):16-26. doi: 10.1016/j.pestbp.2004.09.007.

26. Zabetakis L, Edwards R, O'Hagan D. Elicitation of tropane alkaloid biosynthesis in transformed root cultures of Datura stramonium. Phytochemistry. 1999;50(1):53-56. doi: 10.1016/ s0031-9422(98)00490-7.

27. Herbert RB. The biosynthesis of plant alkaloids and nitrogenous microbial metabolites. Nat Prod Rep. 2001;18(1):50-65. doi: 10.1039/a809393h.

28. Memelink J, Verpoorte R, Kijne JW. ORCAnization of 
jasmonate-responsive gene expression in alkaloid metabolism. Trends Plant Sci. 2001;6(5):212-219. doi: 10.1016/s13601385(01)01924-0.

29. Nultsch W. Botanique générale. 10th ed. De Boeck: UniversitéTheime Verlag, Bruxelles, Belgique; 1998.

30. Kang SM, Jung HY, Kang YM, Yun DJ, Bahk JD, Yang JK, Choi MS. Effects of methyl jasmonate and salicylic acid on the production of tropane alkaloids and the expression of PMT and $\mathrm{H} 6 \mathrm{H}$ in adventitious root cultures of Scopolia parviflora. Plant Sci. 2004;166(3):745-751. doi: 10.1016/j.plantsci.2003.11.022.

31. Gundlach H, Müller MJ, Kutchan TM, Zenk MH. Jasmonic acid is a signal transducer in elicitor-induced plant cell cultures. Proc Natl Acad Sci Unit States Am. 1992;89(6):2389-2393. doi: 10.1073/pnas.89.6.2389.

32. Blechert S, Brodschelm W, Hölder S, Kammerer L, Kutchan TM, Mueller MJ, Xia ZQ, Zenk M.H. The octadecanoid pathway: signal molecules for the regulation of secondary pathways. Proc Natl Acad Sci Unit States Am. 1995;92(10):4099-4105. doi: 10.1073/pnas.92.10.4099.

33. Doares SH, Syrovets T, Weiler EW, Ryan CA. Oligogalacturonides and chitosan activate plant defensive genes through the octadecanoid pathway. Proc Natl Acad Sci Unit States Am. 1995;92(10):4095-4098. doi: 10.1073/ pnas.92.10.4095

34. Wasternack C, Parthier B. Jasmonate-signalled plant gene expression. Trends Plant Sci. 1997;2(8):302-307. doi: 1016/ s1360-1385(97)89952.9

35. Reymond P, Farmer EE. Jasmonate and salicylate as global signals for defense gene expression. Curr Opin Plant Biol. 1998;1(5):404-411. doi: 10.1016/s1369-5266(98)80264-1.

36. Yu KW, Gao WY, Son SH, Paek KY. Improvement of ginsenoside production by jasmonic acid and some other elicitors in hairy root culture of ginseng (Panax ginseng C. A. Meyer). Vitro Cell Dev Biol Plant. 2000;36(5):424-428. doi: 10.1007/s11627-0000077-4.

37. Yu KW, Hahn EJ, Paek KY. Ginsenoside Production by Hairy Root Cultures of Panax ginseng C.A. Meyer in Bioreactors.
Proc Int Conf on MAP Eds. J. Bernáth et al. Acta Hortic. 2003;597:237-243. doi: 10.17660/actahortic.2003.597.34.

38. Ruiz-May E, De-la-Peña C, Galaz-Ávalos RM, Lei Z, Watson BS, Sumner LW, Loyola-Vargas VM. Methyl Jasmonate Induces ATP Biosynthesis Deficiency and Accumulation of Proteins Related to Secondary Metabolism in Catharanthus roseus (L.) G. Hairy Roots. Plant Cell Physiol. 2011;52(8):1401-1421. doi: $10.1093 / \mathrm{pcp} / \mathrm{pcr} 086$.

39. Gamborg OL, Miller RA, Ojima K. Nutrient requirements of suspension cultures of soybean root cells. Exp. Cell Res. 1968;50(1):151-158. doi: 10.1016/0014-4827(68)90403-5.

40. Kartal M, Kurucu S, Altun L, Ceyhan T, Sayar E, Cevheroğlu S, Yetkin Y. Quantitative Analysis of 1-Hyoscyamine in Hyoscyamus reticulatus L. by GC-MS. Turk $J$ Chem. 2003;27:565-569.

41. Goupy J, Creighton L. Introduction aux plans d'expériences. 3rd ed. DUNOD: Paris, France; 2006.

42. Pasandideh SHR, Niaki STA. Multi-response simulation optimization using genetic algorithm within desirability function framework. Appl Math Comput. 2006;175(1):366-382. doi: 10.1016/j.amc.2005.07.023.

43. Baş D, Boyaci IH. Modeling and optimization I: Usability of response surface methodology. J Food Eng. 2007;78(3):836845. doi: 10.1016/j.jfoodeng.2005.11.024.

44. Mason RL, Gunst RF, Hess JL. Statistical Design and Analysis of Experiments with Applications to Engineering and Science. 2nd ed. A John Wiley \& Sons publication: NY, USA.2003. doi: 10.1002/0471458503.

45. Bezerra MA, Santelli RE, Oliveira EP, Villar LS, Escaleira LA. Response surface methodology (RSM) as a tool for optimization in analytical chemistry. Talanta. 2008;76(5):965-977. doi: 10.1016/j.talanta.2008.05.019.

46. Zayed R, Wink M. Induction of tropane alkaloid formation in transformed root cultures of Brugmansia suaveolens (Solanaceae). Z Naturforsch C. 2004;59(11-12):863-867. doi: 10.1515/znc-2004-11-1216. 